\title{
Polychromatic femtosecond fluorescence studies of metal-polypyridine complexes in solution
}

\author{
Olivier Bräm, Fabrizio Messina, Ahmed M. El-Zohry, Andrea Cannizzo ${ }^{1}$, Majed Chergui * \\ Ecole Polytechnique Fédérale de Lausanne, Laboratoire de Spectroscopie Ultrarapide (LSU), ISIC, Faculté des Sciences de Base, Station 6, CH-1015 Lausanne-Dorigny, Switzerland
}

\section{A R T I C L E I N F O}

\section{Article history:}

Received 27 September 2011

In final form 14 November 2011

Available online 3 December 2011

\section{Keywords:}

Metal-polypyridine complexes

IVR

Internal conversion

Intersystem crossing

Ultrafast

Fluorescence up-conversion

\begin{abstract}
A B S T R A C T
Femtosecond-resolved broadband fluorescence studies are reported for $\left[M(b p y)_{3}\right]^{2+}(\mathrm{M}=\mathrm{Fe}$, Ru), RuN3 and RuN719 complexes in solution. We investigated the pump wavelength dependence of the fluorescence of aqueous $\left[\mathrm{Fe}(\mathrm{bpy})_{3}\right]^{2+}$ and the solvent and ligand dependence of the fluorescence of Ru-complexes excited at $400 \mathrm{~nm}$. For all complexes, the ${ }^{1} \mathrm{MLCT}$ fluorescence appears at zero time delay with a mirror-like image with respect to the absorption. It decays in $\leqslant 30-45$ fs due to intersystem crossing to the ${ }^{3}$ MLCT states, but a longer lived component of $\sim 190 \mathrm{fs}$ additionally shows up in RuN719 and RuN3. No solvent effects are detected. The very early dynamics are characterized by internal conversion (IC) and intramolecular vibrational redistribution (IVR) processes on a time scale which we estimate to $\leqslant 10$ fs using the ${ }^{1}$ MLCT lifetime as an internal clock.
\end{abstract}

(ㄷ) 2011 Elsevier B.V. All rights reserved.

\section{Introduction}

Due to their peculiar excited-state dynamics, metal-polypyridine complexes are extensively used in photochemical applications, such as solar energy conversion [1-3]. These complexes, of which ruthenium tris-bipyridine $\left(\left[\mathrm{Ru}(\mathrm{bpy})_{3}\right]^{2+}\right)$ is considered the prototype, exhibit a visible absorption band due to the singlet metal-to-ligand charge transfer $\left({ }^{1} \mathrm{MLCT}\right)$ state. The principle of the dye sensitized solar cells (DSSCs) is based on the use of such metal-based molecular systems, of which the RuN3 ([Ru(dcb$\left.\left.\mathrm{pyH}_{2}\right)_{2}(\mathrm{NCS})_{2}\right]$ ) dye is the most popular, adsorbed onto a semiconductor substrate (usually $\mathrm{TiO}_{2}$ ). Upon excitation of the ${ }^{1}$ MLCT state, injection of an electron into the substrate occurs [4]. A central issue for the efficiency of dye-sensitized solar cells is the detailed understanding of the mechanisms and time scales of electron injection. Much effort has been devoted to this aim in the past 15 years, mainly using nanosecond to femtosecond transient absorption spectroscopy in the visible, near-IR, and mid-IR spectral regions [5-17]. Overall, it was concluded that injection spans several time scales, ranging from a few tens of femtoseconds from the Franck-Condon accessed state to several picoseconds from the ${ }^{3}$ MLCT state, which is populated by intersystem crossing (ISC) from the ${ }^{1}$ MLCT state. A detailed understanding of the initial events at the Franck-Condon state requires a description of the ultrafast

\footnotetext{
* Corresponding author.

E-mail address: Majed.Chergui@epfl.ch (M. Chergui).

${ }^{1}$ Now at Institute of Applied Physics, University of Bern, Sidlerstr. 5, CH-3012 Bern, Switzerland.
}

intramolecular processes occurring in the ${ }^{1}$ MLCT state. For this purpose, ultrafast fluorescence studies have proven very insightful.

Ultrafast time-gated fluorescence is ideal to investigate the evolution of excited molecules because it involves only the ground, the initially excited state and lower lying excited states eventually populated from the latter. On the other hand, transient absorption contains several overlapping contributions due to ground state bleach, excited state absorption and stimulated emission, which sometimes complicate the analysis. Bhasikuttan and Okada reported the first femtosecond fluorescence measurements at single emission wavelengths of the RuN3 dye in ethanol with a resolution of $130 \mathrm{fs}$ [18]. They derived a decay of $\sim 40 \mathrm{fs}$ of the fluorescence at $600 \mathrm{~nm}$, which they attributed to intersystem crossing and internal conversion. They also noted a lengthening of the decay time towards longer emission wavelengths. As pointed out by them, the limited data available under such conditions did not allow getting a full picture of the ultrafast relaxation kinetics. In addition, their laser source ran at a repetition rate of $82 \mathrm{MHz}$ (period $12 \mathrm{~ns}$ ), while the ${ }^{3}$ MLCT lifetime of RuN3 in ethanol is 59 ns [4], which may cause build-up problems due to reexcitation of the excited state by subsequent pulses.

We recently reported polychromatic time-resolved fluorescence studies with a time resolution of $\sim 100$ fs allowing us to record the entire emission profile of the system at a given time delay after excitation [19-21]. We presented results on $\left[\mathrm{Ru}(\mathrm{bpy})_{3}\right]^{2+}[22]$ and $\left[\mathrm{Fe}(\mathrm{bpy})_{3}\right]^{2+}[23]$ in aqueous solutions, detecting not only their short lived ${ }^{1}$ MLCT fluorescence but also their ${ }^{3}$ MLCT phosphorescence. For both complexes, the decay time of the ${ }^{1} \mathrm{MLCT}$ emission was found to be $\leqslant 30 \mathrm{fs}$, and the fluorescence exhibited fairly 
similar features, characterized by a strongly Stokes shifted ${ }^{1}$ MLCT fluorescence already at zero time delay, with a mirror-like profile with respect to the absorption band (Fig. 1). This implies sub$30 \mathrm{fs}$ intramolecular energy relaxation processes preceding the ${ }^{1}$ MLCT fluorescence, such as internal conversion (IC) in the manifold of ${ }^{1}$ MLCT states and intramolecular vibrational redistribution (IVR). Since DSSCs usually operate with RuN3 or RuN719 $\left(\left(\mathrm{Bu}_{4} \mathrm{~N}\right)_{2}\left[\mathrm{Ru}(\mathrm{dcbpyH})_{2}(\mathrm{NCS})_{2}\right]^{2-}\right)$ complexes, we decided to extend our studies to these complexes, while revisiting the $\left[M(b p y)_{3}\right]^{2+}$ complexes to investigate the pump wavelength and solvent dependences of their ultrafast intramolecular relaxation processes. Furthermore, RuN3 and RuN719 are asymmetric complexes contrary to $\left[\mathrm{Ru}(\mathrm{bpy})_{3}\right]^{2+}$, which allows us to explore the effects of molecular geometry on the ultrafast relaxation dynamics of this class of molecules.

\section{Experimental procedures}

The experimental set-up is described in details in Refs. [19-21], and we just present its features of relevance here. Briefly, the sample was either excited by $400 \mathrm{~nm}$ pulses (typical width $60 \mathrm{fs}$, power $80 \mathrm{~nJ} /$ pulse, focal spot $30 \mu \mathrm{m}$ (FWHM), at repetition rates of 150$250 \mathrm{kHz}$ ) or between 480 and $700 \mathrm{~nm}$ from an optical parametric amplifier (OPA) system (Coherent, OPA-9400, typical width $70 \mathrm{fs,}$ power $80 \mathrm{~nJ} /$ pulse, focal spot $30 \mu \mathrm{m}$ (FWHM), at repetition rates of $150-250 \mathrm{kHz}$ ). The emission, collected in forward-scattering geometry, was up-converted in a $250-\mathrm{mm}$ thick $\beta$-barium borate (BBO) crystal by mixing it with a gate pulse at $800 \mathrm{~nm}$. The up-converted signal was spatially filtered and detected with a spectrograph and a liquid- $\mathrm{N}_{2}$-cooled charge-coupled device (CCD) camera in polychromatic mode. Appropriate Schott filters (Schott Glass Technologies) were used to attenuate the remaining excitation light. This greatly improved the signal-to-noise ratio but limited the detectable spectral range to a region starting $40 \mathrm{~nm}$ after the excitation wavelength up to $690 \mathrm{~nm}$. The time resolution of the setup is $\sim 100 \mathrm{fs}$, as measured by the FWHM of a kinetic trace of the Raman line of $\mathrm{H}_{2} \mathrm{O}$.

Tris(2,2'-bipyridine)iron(II) chloride hexahydrate ([Fe ${ }^{\mathrm{II}}\left(2,2^{\prime}-\right.$ bipyridine $\left.)_{3}\right] \mathrm{Cl}_{2} \times 6 \mathrm{H}_{2} \mathrm{O}$ ) was synthesized according to Ref. [24] and dissolved in deionized water. The dyes RuN3 ([Ru(dcb$\left.\left.\left.\mathrm{pyH}_{2}\right)_{2}(\mathrm{NCS})_{2}\right]\right)$ and RuN719 $\left(\left(\mathrm{Bu}_{4} \mathrm{~N}\right)_{2}\left[\mathrm{Ru}(\mathrm{dcbpyH})_{2}(\mathrm{NCS})_{2}\right]^{2-}\right)$ $\left[\mathrm{dcbpy}=4,4^{\prime}\right.$-dicarboxy-2,2'-bipyridine] were synthesized

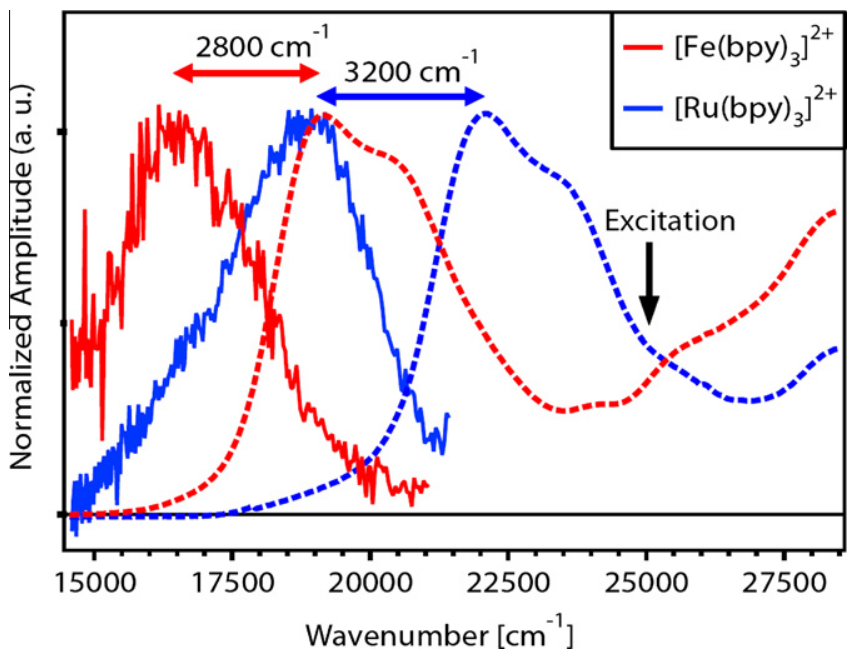

Fig. 1. Steady-state absorption spectra showing the ${ }^{1}$ MLCT absorption band (dashed traces) and time-zero fluorescence spectra of $\left[\mathrm{Fe}(\mathrm{bpy})_{3}\right]^{2+}$ and $\left[\mathrm{Ru}(\mathrm{bpy})_{3}\right]^{2+}$ in water, excited at $25,000 \mathrm{~cm}^{-1}$ (black arrow). The horizontal arrows indicate the respective absorption-emission Stokes shift (from Refs. [22,23]). according to Ref. [25] and dissolved in ethanol, acetonitrile and water at two different pH's (5.4 and 7). The solutions, at room temperature, were circulated in a $0.2 \mathrm{~mm}$ thick quartz flow-cell at a speed of $1 \mathrm{~m} / \mathrm{s}$ to avoid photo-damage. With the above experimental conditions, the excitation pulses hit the same spot $\sim 8$ times at $250 \mathrm{kHz}$. However, since the ground state recovery is several tens to hundreds of ns, all excited molecules relax to the ground state between two pulses. The concentration was chosen to have typically between $0.1 \mathrm{OD}$ and $0.3 \mathrm{OD}$ at the excitation wavelength Typical data acquisition times for all up-conversion measurements were of the order of half an hour for one scan, the scans were repeated up to 10 times and then averaged.

The data were analyzed by performing a global fit (GF) of kinetic traces averaged over $5 \mathrm{~nm}$ steps using Eq. (1):

$I=\left\{A_{1} \cdot e^{\left(-t / \tau_{1}\right)}+A_{2} \cdot e^{\left(-t / \tau_{2}\right)}\right\} \otimes e^{\left[-\left(\frac{t-t_{0}}{\Delta_{\text {IRF }} /(2 \sqrt{\ln 2})}\right)^{2}\right]}$

in which we assume two characteristic times $\left(\tau_{1}, \tau_{2}\right)$ for the emission decay. The Gaussian term describes the convolution with the instrument response function (IRF), where $\Delta_{I R F}$ and $t_{0}$ are its fwhm and the time zero, respectively. In the GF procedure, the time constants have been considered as common kinetic parameters at all wavelengths, whereas the amplitudes $A_{1}$ and $A_{2}$ have been determined for each wavelength. In order to reconstruct the timeintegrated spectrum associated to each time constant obtained, we multiplied the pre-exponential factors $A_{1}$ and $A_{2}$ by the respective decay times and plotted them as a function of wavelength.

\section{Results and discussion}

Fig. 1 shows the steady state absorption spectra and the previously reported fluorescence spectra at zero time delay of the $[\mathrm{Ru}(\mathrm{b}-$ py $\left.)_{3}\right]^{2+}$ and $\left[\mathrm{Fe}(\mathrm{bpy})_{3}\right]^{2+}$ complexes $[22,23]$. The steady-state absorption shows some structures, which is due to a vibrational progression of the high frequency Franck-Condon (FC) mode at $1607 \mathrm{~cm}^{-1}$ (see also Fig. 2). This progression was more clearly observed in low temperature spectra of single crystals of $\left[\mathrm{Ru}(\mathrm{bpy})_{3}\right]^{2+}$ $[26]$ or of $\left[\mathrm{Fe}(\mathrm{bpy})_{3}\right]^{2+}$ doped in inert $\left[\mathrm{Zn}(\mathrm{bpy})_{3}\right]^{2+}$ matrices [27]. Furthermore, for $\left[\mathrm{Ru}(\mathrm{bpy})_{3}\right]^{2+}$ at low temperatures the main progression contains satellite bands, and additional progressions set

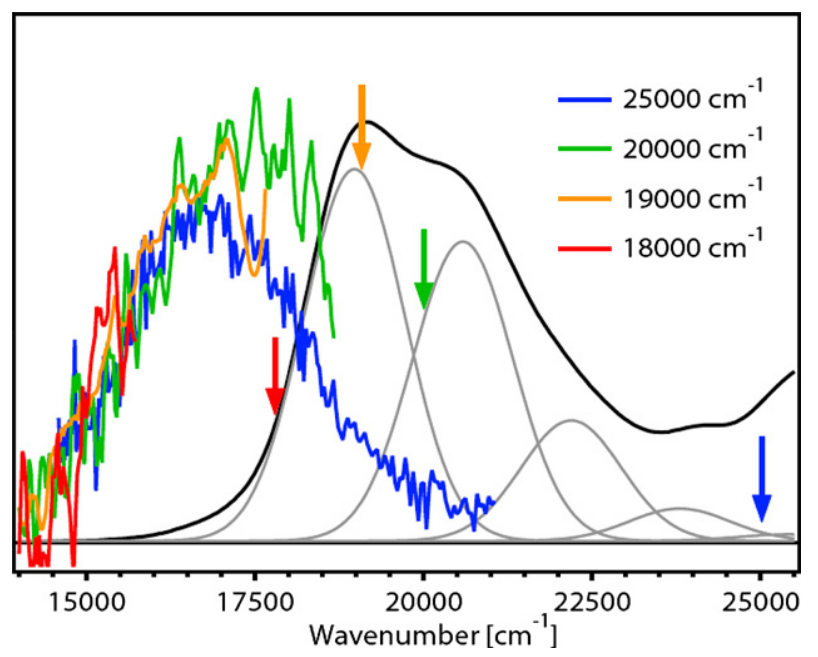

Fig. 2. Ground state absorption of $\left[\mathrm{Fe}(\mathrm{bpy})_{3}\right]^{2+}$ in water (black trace) with its decomposition in terms of a progression of the Franck-Condon $1607 \mathrm{~cm}^{-1}$ vibrational mode (grey lines) [23]. The coloured traces show the time-zero emission spectra for different excitation wavelengths (marked by vertical arrows with the respective colours). The emission spectra are cut-off on the blue side according to the corresponding filter used to reject excitation light. (For interpretation of the references to colour in this figure legend, the reader is referred to the web version of this article.) 


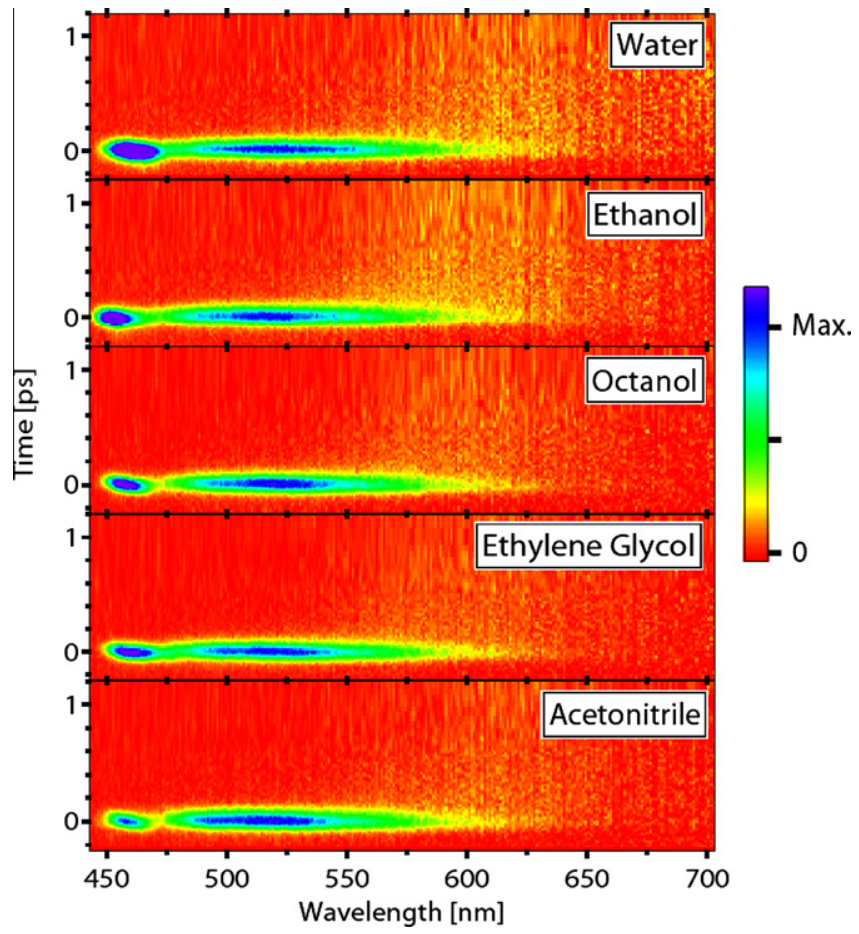

Fig. 3. $2 \mathrm{D}$ time-wavelength plots of the emission of $\left[\mathrm{Ru}(\mathrm{bpy})_{3}\right]^{2+}$ excited at $400 \mathrm{~nm}$ in different solvents. The plots are normalized to the maximum of the fluorescence $(\sim 520 \mathrm{~nm})$

above $23,000 \mathrm{~cm}^{-1}$, due to higher electronic states [26]. The same Franck-Condon progression is observed for $\left[\mathrm{Fe}(\mathrm{bpy})_{3}\right]^{2+}$, but not as clearly as in the case of $\left[\mathrm{Ru}(\mathrm{bpy})_{3}\right]^{2+}$ because of a congestion of the spectrum by underlying contributions. Indeed, in both cases, polarization dependent absorption spectra show that there are several states absorbing in the region of the ${ }^{1}$ MLCT state and in particular, in the $400 \mathrm{~nm}$ region [26-28]. Finally, the low energy tail of the absorption band is clearly due to a weak absorption of the ${ }^{3}$ MLCT states (below 20,000 $\mathrm{cm}^{-1}$ for the $\mathrm{Ru}$ complex [28] and $17,500 \mathrm{~cm}^{-1}$ for the Fe one).

In Fig. 1, the time zero fluorescence exhibits a quite similar absorption-emission Stokes shift for both complexes with, as already mentioned, a near-mirror image with respect to the absorption spectrum, as expected for a vibrationally cold emission, despite the fact that we excite high vibrational levels ( $v=2$ and 4 , respectively) of the high frequency $1607 \mathrm{~cm}^{-1}$ mode, which makes up the dominant progression of the absorption band (Fig. 2), as well as higher lying electronic states. To further clarify this behaviour, we measured the fluorescence spectra at $t=0$ as a function of excitation energy, thus allowing us to deposit different amounts of excess vibrational energy in the excited electronic state. This was only possible for $\left[\mathrm{Fe}(\mathrm{bpy})_{3}\right]^{2+}$ because its absorption band best matches the range of excitation wavelengths available with our set-up.

Fig. 2 shows the absorption spectrum of aqueous $\left[\mathrm{Fe}(\mathrm{bpy})_{3}\right]^{2+}$ along with its decomposition [23] using the progression of the high frequency Franck-Condon (FC) mode at $1607 \mathrm{~cm}^{-1}$ with a HuangRhys factor $S=0.8$, whose bands are represented by Gaussian line shapes. As already mentioned this description of the absorption band is not complete as it overlooks the fact that other electronic states make it up, but it is a useful starting point for the discussion of our observations. The different pump wavelengths used to excite the system are indicated by arrows. The highest excitation frequency used here is close to the position of the $v=4$ vibrational level of the FC mode, while the others excite the $v=0$ and $v=1$
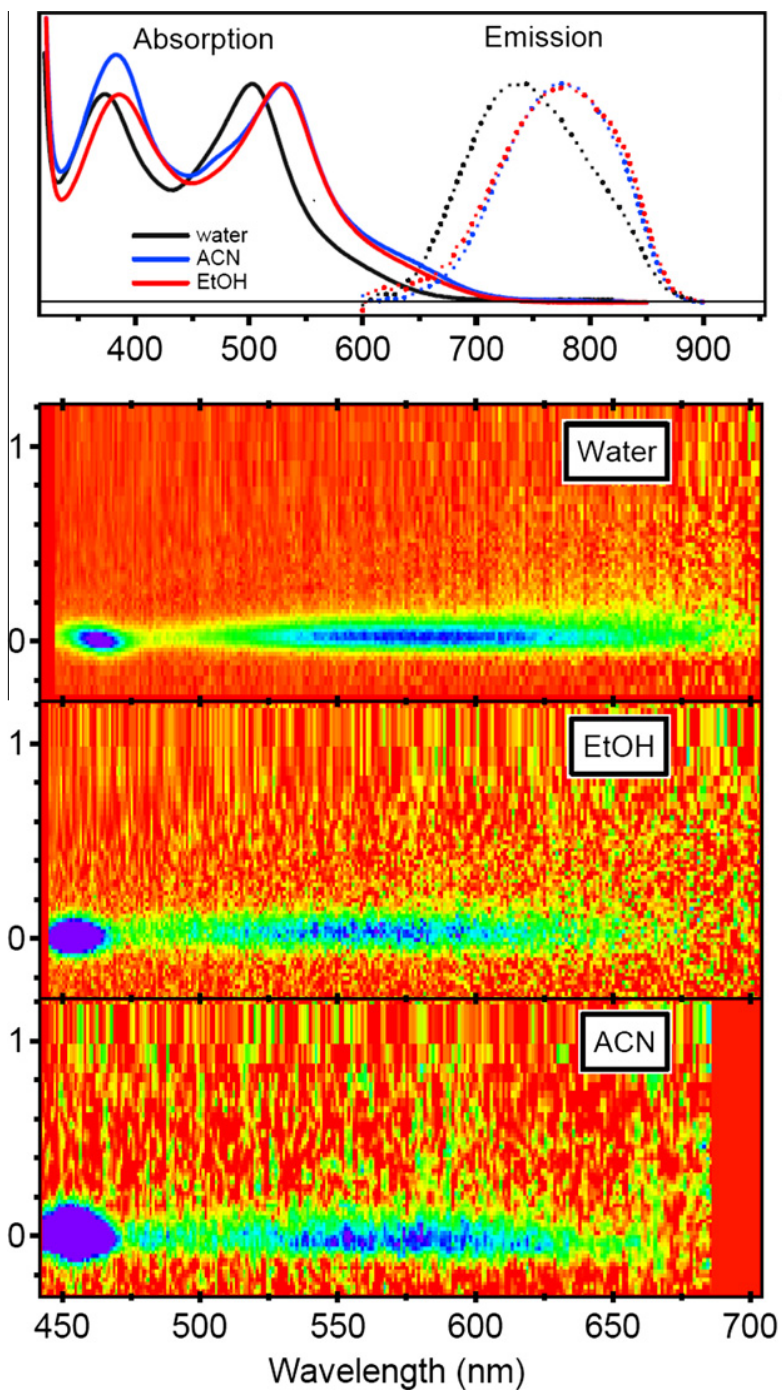

Fig. 4. Top panel: Static absorption and emission spectra of RuN719 in different solvents. Lower panels: 2D time-wavelength plots of the emission of RuN719 excited at $400 \mathrm{~nm}$ in several solvents normalized to the maximum of the fluorescence $(\sim 570 \mathrm{~nm})$

levels. The corresponding emission spectra at $t=0$ are also shown. Over the detection range allowed by the corresponding excitation cut-off filter, they clearly show no excitation wavelength dependence. Also to note is the slight disagreement that is observed around $18,000 \mathrm{~cm}^{-1}$ where partial re-absorption (sample-dependent, but always $\leqslant 25 \%$ ) of the emitted fluorescence occurs due to the red wing of the absorption band. In all cases the decay of the ${ }^{1}$ MLCT fluorescence is found to occur in $\leqslant 30 \mathrm{fs}$, as previously reported under $400 \mathrm{~nm}$ excitation and shown to be due to an ultrafast intersystem crossing to the ${ }^{3}$ MLCT state [23]. Note that the mirror symmetry of the $t=0$ emission for $\left[\mathrm{Ru}(\mathrm{bpy})_{3}\right]^{2+}$ (Fig. 1 ) is also consistent with the lack of excitation wavelength dependence seen in Fig. 2, since the $400 \mathrm{~nm}$ excitation corresponds to the $v=2$ level in the former. Together with Fig. 1, this confirms that the ${ }^{1}$ MLCT fluorescence is vibrationally relaxed on ultrashort time scales. This implies that the time scale of such a relaxation must be significantly shorter than $30 \mathrm{fs}$. We stress that the concept of a "cold" ${ }^{1}$ MLCT fluorescence is with respect to the high frequency Franck-Condon mode making up the main progression in absorption (Fig. 2), but the excess energy is redistributed in lower frequency modes. 


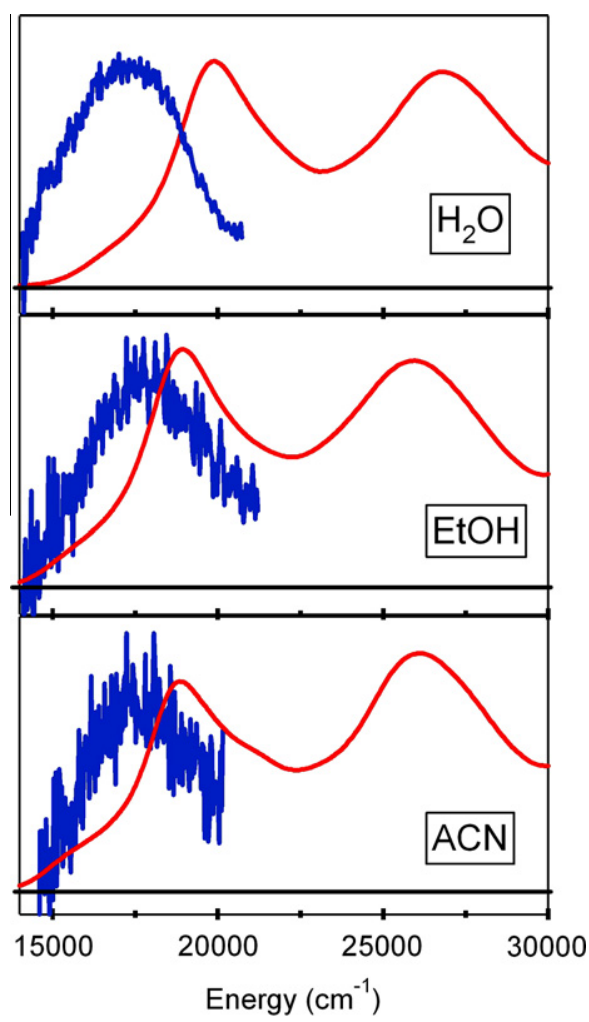

Fig. 5. Steady-state absorption spectra of RuN719 in different solvents (red traces) and time gated fluorescence recorded at zero time delay (blue traces). The samples were excited at $400 \mathrm{~nm}$. (For interpretation of the references to colour in this figure legend, the reader is referred to the web version of this article.)

Starting from the fact that the ${ }^{1}$ MLCT fluorescence exhibits an upper lifetime $\tau_{\text {ISC }} \sim 30$ fs, determined by the intersystem crossing (ISC) to the ${ }^{3}$ MLCT state both in $\left[\mathrm{Ru}(\mathrm{bpy})_{3}\right]^{2+}$ and $\left[\mathrm{Fe}(\mathrm{bpy})_{3}\right]^{2+}$ $[22,23]$, one can roughly estimate the relaxation time $\tau_{\mathrm{H}}$ (H for hot) leading to the observed "cold" signal from the ratio $R$ of the peak fluorescence signal to that of the background on the blue most side of the spectrum, namely $\tau_{\mathrm{H}} \sim \mathrm{R}^{-1} \tau_{\text {ISC. }}$. To this purpose, let us consider the case of $400 \mathrm{~nm}$ excitation of $\left[\mathrm{Fe}(\mathrm{bpy})_{3}\right]^{2+}$. In first approximation, this will basically populate only the ${ }^{1}$ MLCT $v=4$ level and the underlying higher electronic states. In the absence of any relaxation, the initially prepared state(s) should yield a characteristic vibrationally hot emission $I_{\mathrm{H}}(E)$ arising from the $v=4$ level to the vibrational levels of the ground state, as well as emission from higher lying electronic states. By calculating the shape of $I_{\mathrm{H}}(E)$ for the case of the $v=4$ emission via the known Frank-Condon parameters of $\left[\mathrm{Fe}(\mathrm{bpy})_{3}\right]^{2+}[23]$ it turns out that most of the $I_{\mathrm{H}}(E)$ emission intensity ( 65\%) is concentrated in the "hot" region $E_{\mathrm{H}}>19,000 \mathrm{~cm}^{-1}$, higher than the centre of gravity of our signal. Hence, to be consistent with the observed Stokes-shifted fluorescence, such an initially prepared hot state must be (incoherently) depopulated in a time scale $\tau_{\mathrm{H}}$, leading to a "cold" (C) emitting state with emission $I_{C}(E)$ that subsequently decays in $\tau_{\text {ISc }}$. Since both $\tau_{\text {ISC }}$ and $\tau_{\mathrm{H}}$ are significantly shorter than the $100 \mathrm{fs}$ IRF, the dynamics is pulse-limited, meaning that time-zero populations of $\mathrm{H}$ and $\mathrm{C}$ will be basically proportional to $\tau_{\mathrm{H}}$ and $\tau_{\text {ISC }}$, respectively. We can thus express the normalized fluorescence line shape as a weighted combination of hot and cold emission line shapes: $I(E)=\left(\tau_{\mathrm{H}}+\tau_{\text {ISC }}\right)^{-1}\left[\tau_{\mathrm{H}} I_{\mathrm{H}}(E)+\tau_{\text {ISC }} I_{\mathrm{C}}(E)\right]$, namely $\tau_{\mathrm{H}} /\left(\tau_{\mathrm{H}}+\tau_{\text {ISC }}\right)$ corresponds to the weight of the "hot" contribution to the whole line shape. Since $I_{\mathrm{H}}(E)$ is negligible in the region $E_{\mathrm{C}}$ where the signal peaks, and since $I_{\mathrm{C}}(E)$ is negligible in the $E_{\mathrm{H}}$ energy region, the last expression implies that $\tau_{\mathrm{H}} \sim \mathrm{R}^{-1} \tau_{\text {ISC }}$ within a factor of the order of unity (basically corresponding to the width ratio between the line shapes $I_{\mathrm{C}}(E)$ and $I_{\mathrm{H}}(E)$ ).

The ratio $R$ can be assessed by comparing in Fig. 2 the signal at $16,500 \mathrm{~cm}^{-1}$ to that at $20,000-21,000 \mathrm{~cm}^{-1}$ for the spectrum excited at $25,000 \mathrm{~cm}^{-1}$, yielding $R \sim 10: 1$ (it is worth noting that reabsorption effects are negligible for this sample both at $E_{\mathrm{H}}$ and $E_{\mathrm{C}}$ ). This implies a lifetime of $<3$ fs for the intermediate states lying between the excitation energy and the fluorescence. Since relaxation is a cascade among intermediate states, we conservatively estimate to $\leqslant 10$ fs the appearance of the Stokes shifted ${ }^{1}$ MLCT fluorescence in $\left[M(b p y)_{3}\right]^{2+}(\mathrm{M}=\mathrm{Ru}, \mathrm{Fe})$. Although we used the vibrationally hot emission in the above estimate, the arguments are perfectly valid for emission from a higher electronic state. This is actually fully supported by the results below on the RuN719 dye. The estimate implies that the "cold" ${ }^{1}$ MLCT emission is formed on sub-vibrational time scales, which can only be due to ultrafast IC and IVR [29]. In support of this, we note that the vibrational progression of the highest frequency mode $\left(1607 \mathrm{~cm}^{-1}\right)$ dominating the ${ }^{1} \mathrm{MLCT}$ absorption band of $\left[M(b p y)_{3}\right]^{2+}(\mathrm{M}=\mathrm{Ru}, \mathrm{Fe})$ features a line width of $\sim 1700 \mathrm{~cm}^{-1}$ of each vibronic line [22,23]. At cryogenic temperatures, the line width was observed to be 400 $500 \mathrm{~cm}^{-1}$ [26], which would correspond to a lifetime of $10-15 \mathrm{fs}$, assuming a fully homogenous lifetime broadening mechanism. While the observed ${ }^{1}$ MLCT fluorescence stems from a "cold" electronic level with respect to the high frequency Franck-Condon mode, we remark again that energy has been redistributed among optically silent lower frequency modes, since cooling of the molecule by the solvent is unlikely to occur within such a short time scale. The high local temperature of the molecule after IVR should lead to a structureless fluorescence at early times, as opposed to the vibrational structure that shows up in absorption. Such a broadening of bands at time zero and their narrowing at later times was for example clearly visible in our fluorescence up-conversion study of the 2,5-diphenyloxazole (PPO) dye in cyclohexane $[21,30]$. This does not occur here because the ${ }^{1}$ MLCT lifetime is too short to allow for the manifestation of cooling.

The above estimate of $\leqslant 10 \mathrm{fs}$ for the dynamical Stokes shift is significantly shorter than any solvation times [31]. However, given the measurable solvent effects on the absorption spectrum (see e.g., Ref. [32] for the case of $\left[\mathrm{Ru}(\mathrm{bpy})_{3}\right]^{2+}$ ), we nevertheless explored their possible influence in the initial intramolecular relaxation. Fig. 3 shows the 2D time-wavelength plots of the emission of $\left[\mathrm{Ru}(\mathrm{bpy})_{3}\right]^{2+}$ excited at $400 \mathrm{~nm}$ in various solvents. They all feature a short-lived singlet emission band centred around $500-525 \mathrm{~nm}$, and a longer lived ${ }^{3}$ MLCT emission centred around $600-650 \mathrm{~nm}$, as previously reported [22]. Except for minor spectral shifts between the different solvents, their dynamics are clearly identical. Given that the used solvents differ significantly in their dielectric constants, we can safely conclude that the dynamics at ultrashort times is entirely governed by intramolecular processes. Note that, as previously reported for the water solvent [22], the ${ }^{3}$ MLCT phosphorescence is already within the first $100 \mathrm{fs}$ at its steady state energy in all solvents.

Because RuN3 and RuN719 are asymmetric compared to [Ru(bpy $\left.)_{3}\right]^{2+}$, we explored the effects of molecular geometry on the intramolecular dynamics of metal-polypyridine complexes, focusing on RuN719. The static absorption spectrum of RuN719 features two ${ }^{1}$ MLCT bands (Fig. 4) located at 500-530 nm and 375-385 nm. Excitation into these bands leads to the steady-state phosphorescence spectrum that peaks at 740-780 nm (Fig. 4). Both absorption and phosphorescence spectra exhibit clear solvent effects with ethanol and acetonitrile having rather similar features while water clearly stands out. This was analyzed by Fantacci et al. [33] using a combined Density Functional/Time Dependent Density Functional approach. The blue shift in water compared to ethanol was related to a decreased dipole moment in the excited state. 


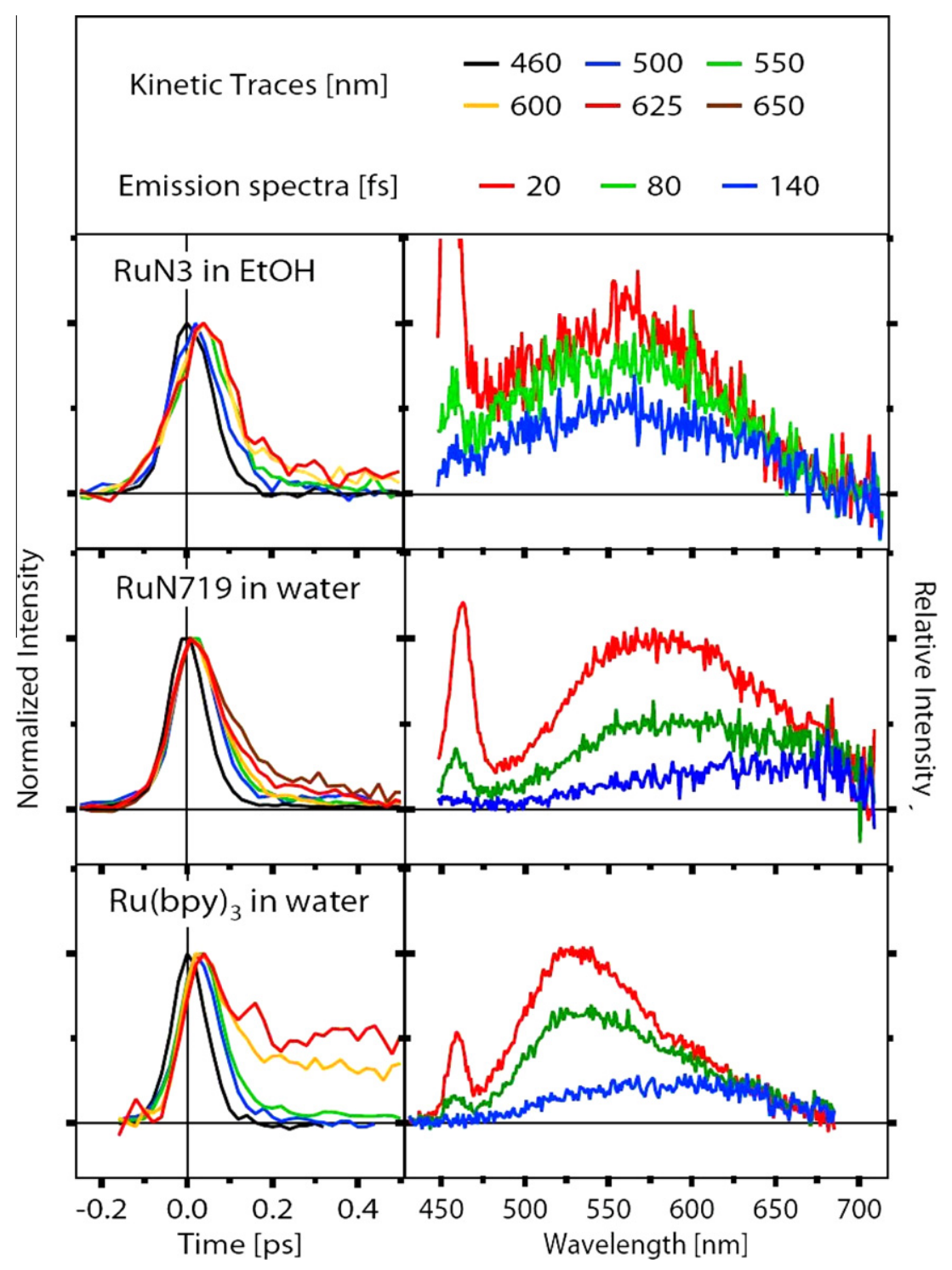

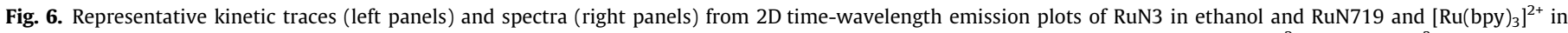

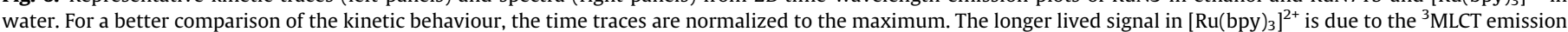

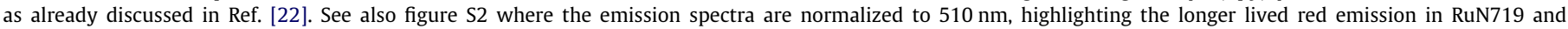
$\left[\mathrm{Ru}(\mathrm{bpy})_{3}\right]^{2+}$. (For interpretation of the references to colour in this figure legend, the reader is referred to the web version of this article.)

The time-wavelength plots of the ultrafast fluorescence of N719 in water, ethanol and acetonitrile excited at $400 \mathrm{~nm}$ are also shown in Fig. 4. Because of the fast photo-aggregation on the walls of the flow cell, the integration time used in EtOH and ACN was shorter than in $\mathrm{H}_{2} \mathrm{O}$, leading to a poorer signal to noise ratio. The behaviour of RuN719 appears to be qualitatively identical to what is observed above in $\left[\mathrm{Ru}(\text { bpy })_{3}\right]^{2+}$ (Fig. 3): the dominant signal in all solvents is the fast-decaying pulse-limited fluorescence around $550-575 \mathrm{~nm}$. The ${ }^{3}$ MLCT phosphorescence is not measured in this case since it falls outside our detection region. Fig. 5 shows the static absorption spectra and the time-zero fluorescence spectrum on the same energy scale. The latter is strongly Stokes-shifted and mirror symmetric with respect to the first absorption band around 18,000 $20,000 \mathrm{~cm}^{-1}$. This points to an ultrafast internal conversion (IC) from the upper to the lower ${ }^{1}$ MLCT state, followed by ultrafast IVR in the latter. Both IC and IVR takes place on a time scale significantly shorter than the fluorescence lifetime ( $\leqslant 30-45 \mathrm{fs}$, see below). Overall, these results (and those for RuN3 in EtOH, not shown here) infer that also for RuN719 and RuN3 the short time dynamics is dominated by intramolecular relaxation (IVR/IC) faster than ISC and that fluorescence arises from a "cold" state. In conclusion, IVR/IC processes occur on similar ultrafast time scales for all Ru-complexes studied here, suggesting a weak influence of the nature and symmetry properties of the ligands coordinating to the central atom.

More information on the decay kinetics of RuN719 and RuN3 can be obtained by a detailed analysis of their kinetic traces. In Fig. 6, we show these for different emission wavelengths and the time-gated emission spectra at different time delays of [Ru(bpy) $\left.]_{3}\right]^{2+}$, RuN719 and RuN3, which are extracted from 2D timewavelength emission plots (i.e., those of Fig. 4 for RuN719, and of Ref. [22] for $\left.\left[\mathrm{Ru}(\mathrm{bpy})_{3}\right]^{2+}\right)$. These traces include the solvent Raman line in the region around $460 \mathrm{~nm}$, which serves as a cross-correlator. It can be seen that the kinetic traces on the blue wing of the fluorescence overlap quite well that of the Raman line, but exhibit a longer decay than the latter, as already reported for [ $\mathrm{Ru}(\mathrm{b}-$ py $\left.)_{3}\right]^{2+}[22]$ and shown to be due to the ultrafast decay (in $\leqslant 30 \mathrm{fs}$ ) of the ${ }^{1}$ MLCT state by intersystem crossing to the ${ }^{3}$ MLCT state. The overall behaviour of RuN3 and RuN719 appears to be quite similar. By convoluting with the cross-correlation time of our experiment 


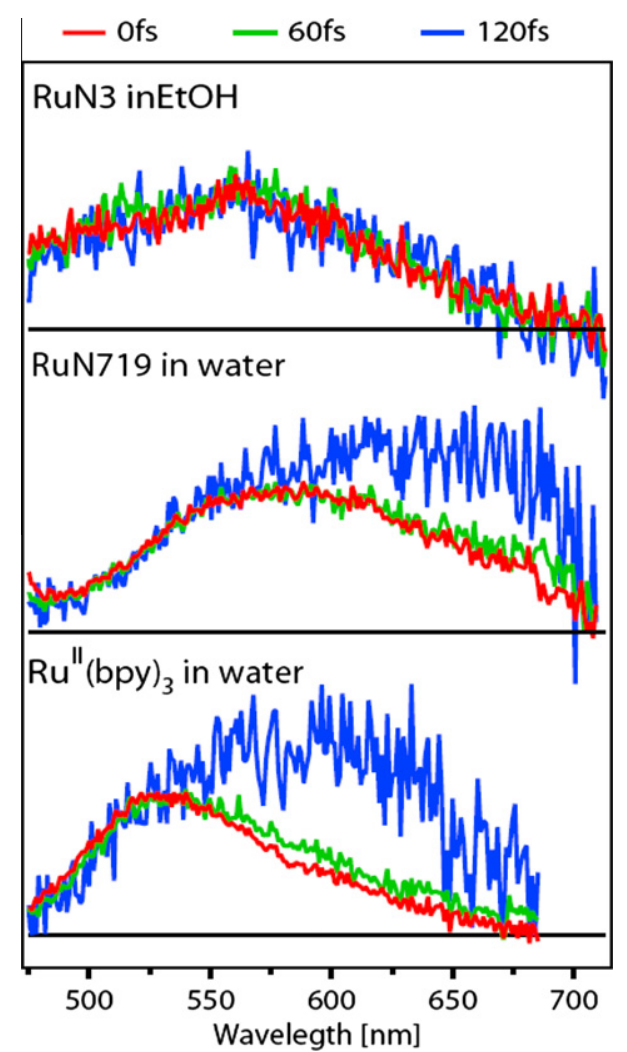

Fig. 7. Representative spectra of emission intensity of RuN3 in ethanol, RuN719 in water and aqueous $\left[\mathrm{Ru}(\mathrm{bpy})_{3}\right]^{2+}$, normalized at $525 \mathrm{~nm}$, where the fluorescence dominates. This normalization shows the increase with time of longer lived ${ }^{1}$ MLCT states emission for RuN719, and of the phosphorescence contribution for $\left[\mathrm{Ru}(\mathrm{bpy})_{3}\right]^{2+}$.

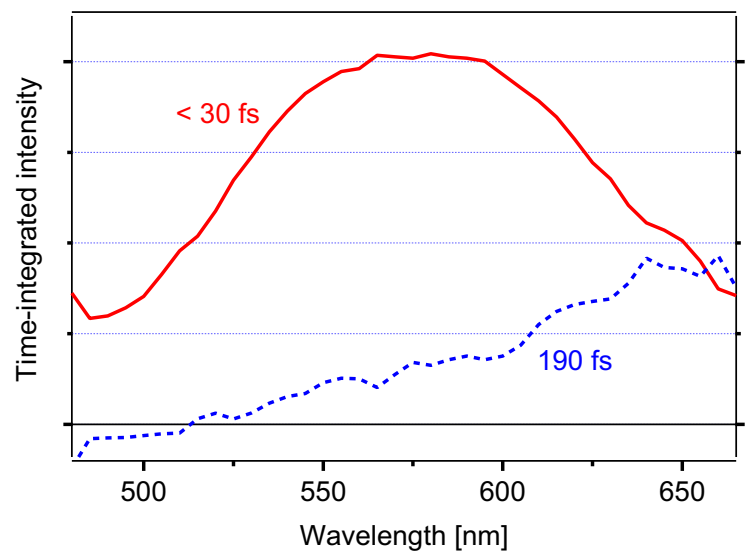

Fig. 8. Time-integrated reconstructed spectra (pre-exponential factor multiplied by time constant, plotted as a function of the emission wavelength) obtained with a global fit analysis of data for RuN719 in water reported in Fig. 4. The uncertainty on the lifetimes of the long component is estimated to be $\pm 30 \mathrm{fs}$.

( $\sim 110 \mathrm{fs}$ ), fits of the kinetic traces at $500 \mathrm{~nm}$ (corresponding to the range of the ${ }^{1} \mathrm{MLCT}$ emission free from longer-lived contributions, see Fig. 7) yield decay times of $\leqslant 30$ fs for RuN719 and $\leqslant 45$ fs for RuN3, in good agreement with Ref. [18]. However, in all three dyes the decay becomes longer for longer detection wavelengths. For example, in the case of RuN3 the fit gives a time constant of $\sim 60$ fs for the trace at $600 \mathrm{~nm}$, wavelength at which Sundstrom and co-workers [13] reported a $\sim 70$ fs lived stimulated emission signal in their transient absorption studies. As this value does not reflect the lifetime of the ${ }^{1}$ MLCT at maximum, it may be due to longer-lived lower lying ${ }^{1}$ MLCT states or a weak contamination by the ${ }^{3}$ MLCT emission, which for RuN3 is peaked at $\sim 800 \mathrm{~nm}$ [18]. For reasons discussed below, we believe that the first option is more likely.

To further investigate the nature of this longer component in RuN719 and RuN3, we performed a global fit analysis of the 2D time-wavelength plot of RuN719 in water, which was chosen because it has the best signal to noise ratio. The result yields two different decay times, corresponding to different spectral components shown in Fig. 8. The first, centred around $570 \mathrm{~nm}$, is pulse-limited ( $\leqslant 30 \mathrm{fs}$ ) and is clearly associated with the ${ }^{1}$ MLCT fluorescence. The second component has a longer decay time ( $\sim 190 \mathrm{fs})$ and lies further to the red, but it does not occur in the range where the ${ }^{3}$ MLCT phosphorescence is expected (compare with top of Fig. 4). It is most likely due to other states of the ${ }^{1}$ MLCT manifold, which have been predicted by Density functional theory (DFT) and timedependent density functional theory (TD-DFT) calculations of RuN3 [33,34]. The analogues of these states for RuN719 may be responsible for this emission.

The results of Figs. 1-3 and 5 clearly show that the singlet emission bands of both Ru and Fe complexes stem from a vibrationally cold state as far as the high frequency Franck-Condon modes are concerned, even though high lying vibrational levels and electronic states are excited. Since the time-zero emission spectra exhibit mirror image symmetry with respect to the absorption band and show no solvent dependence, we can exclude solvation dynamics as being responsible for this behaviour. That the Stokes shift occurs on such an ultrashort time scale, which we estimate to $\leqslant 10$ fs for $\left[M(b p y)_{3}\right]^{2+}$, is quite remarkable given the large amount of energy it represents, and the fact that we are dealing with sub-vibrational time scales, since the highest frequency optically coupled (FC) mode has a period of $\sim 20 \mathrm{fs}\left(1607 \mathrm{~cm}^{-1}\right)$. As a matter of fact, if the system is thought of as a damped classical oscillator, the 10 fs decay time would be associated with a damping ratio as high as $\sim 0.2$, corresponding to $\sim 90 \%$ energy loss within the first oscillation.

As already discussed, the appearance of a mirror-like (with respect to absorption) fluorescence at $t=0$ is due to IC and IVR processes causing an ultrafast departure from the initially excited vibronic level(s). This occurs in the same fashion in Fe- and Rucomplexes and, for the latter, it is influenced neither by the ligands, nor by the solvent. Furthermore, this behaviour appears to be quite general, since it has been observed in other metal complexes $[35,36]$, in the case of organic dyes [30,37-39], and even of chromophores in proteins $[19,40]$. The ultrafast IC may involve conical intersection among the manifold of electronic states, but also and as mentioned above, the excess energy is redistributed among lower frequency modes that are optically silent. Finally, the ultrafast relaxation down the manifold of ${ }^{1}$ MLCT states repeats itself in the ${ }^{3}$ MLCT manifold after the intersystem crossing as witnessed by the fact that the phosphorescence of $\left[\mathrm{Ru}(\mathrm{bpy})_{3}\right]^{2+}$ shows up within 100 fs or so at the same position as in the steady-state spectra [22].

The presence of a weak ${ }^{3}$ MLCT absorption on the red edge of the ${ }^{1}$ MLCT absorption (Figs. 1 and 4), and the fact that the latter has an absorption coefficient that is one order of magnitude smaller that of organic dyes with pure singlet states, suggest that the singlet and triplet MLCT states are strongly mixed. Yet, it is quite remarkable how clearly distinguishable the bands are that are associated to these two classes of states in all emission spectra. It is possible that the IC occurs among the manifold of spin-mixed MLCT states and that part of the population ends up at the bottom of the predominantly ${ }^{1}$ MLCT manifold, while the rest is already in the 
predominantly ${ }^{3}$ MLCT manifold, although the previous $[22,23]$ and present results seem to hint to a relaxation cascade that first takes place among predominantly ${ }^{1}$ MLCT states and then intersystem crosses to the ${ }^{3}$ MLCT manifold.

The above observations have a direct incidence on the functioning of DSSCs, since injection from the Franck-Condon accessed state $[16,17]$ must compete with the ultrafast departure from it due to intramolecular processes such as IC and IVR on a sub-10 fs time scale. This aspect is discussed in a forthcoming paper presenting fluorescence up-conversion studies of DSSCs [41].

\section{Acknowledgments}

We thank Dr M.K. Nazeeruddin for providing us with RuN3 and RuN719 samples and for useful discussions. This work was supported by the Swiss NSF via Grants 200021-107956 and 200021105239.

\section{References}

[1] K. Kalyanasundaram, Coordin. Chem. Rev. 46 (1982) 159.

[2] V. Balzani, A. Juris, M. Venturi, S. Campagna, S. Serroni, Chem. Rev. 96 (1996) 759

[3] V. Balzani, S. Campagna, G. Denti, A. Juris, S. Serroni, M. Venturi, Acc. Chem. Res. 31 (1998) 26.

[4] M.K. Nazeeruddin, A. Kay, I. Rodicio, R. Humphrybaker, E. Muller, P. Liska, N. Vlachopoulos, M. Gratzel, J. Am. Chem. Soc. 115 (1993) 6382.

[5] Y. Tachibana, J.E. Moser, M. Gratzel, D.R. Klug, J.R. Durrant, J. Phys. Chem. 100 (1996) 20056.

[6] T. Hannappel, B. Burfeindt, W. Storck, F. Willig, J. Phys. Chem. B 101 (1997) 6799.

[7] J.E. Moser, D. Noukakis, U. Bach, Y. Tachibana, D.R. Klug, J.R. Durrant, R. Humphry-Baker, M. Gratzel, J. Phys. Chem. B 102 (1998) 3649.

[8] T. Hannappel, C. Zimmermann, B. Meissner, B. Burfeindt, W. Storck, F. Willig, J. Phys. Chem. B 102 (1998) 3651.

[9] R.J. Ellingson, J.B. Asbury, S. Ferrere, H.N. Ghosh, J.R. Sprague, T.Q. Lian, A.J Nozik, J. Phys. Chem. B 102 (1998) 6455.

[10] J.B. Asbury, R.J. Ellingson, H.N. Ghosh, S. Ferrere, A.J. Nozik, T.Q. Lian, J. Phys Chem. B 103 (1999) 3110

[11] J.R. Durrant, Y. Tachibana, I. Mercer, J.E. Moser, M. Gratzel, D.R. Klug, Z. Phys Chem. - Int. J. Res. Phys. Chem. Chem. Phys. 212 (1999) 93.

[12] T.A. Heimer, E.J. Heilweil, C.A. Bignozzi, G.J. Meyer, J. Phys. Chem. A 104 (2000) 4256.

[13] G. Benko, J. Kallioinen, J.E.I. Korppi-Tommola, A.P. Yartsev, V. Sundstrom, J. Am Chem. Soc. 124 (2002) 489.
[14] S.A. Haque, Y. Tachibana, R.L. Willis, J.E. Moser, M. Gratzel, D.R. Klug, J.R. Durrant, J. Phys. Chem. B 104 (2000) 538.

[15] G. Benko, J. Kallioinen, P. Myllyperkio, F. Trif, J.E.I. Korppi-Tommola, A.P. Yartsev, V. Sundstrom, J. Phys. Chem. B 108 (2004) 2862.

[16] G. Benko, P. Myllyperkio, J. Pan, A.P. Yartsev, V. Sundstrom, J. Am. Chem. Soc. 125 (2003) 1118

[17] J. Kallioinen, G. Benko, V. Sundstrom, J.E.I. Korppi-Tommola, A.P. Yartsev, J. Phys. Chem. B 106 (2002) 4396.

[18] A.C. Bhasikuttan, T. Okada, J. Phys. Chem. B 108 (2004) 12629.

[19] M. Vengris, M.A. van der Horst, G. Zgrablic, I.H.M. van Stokkum, S. Haacke, M. Chergui, K.J. Hellingwerf, R. van Grondelle, D.S. Larsen, Biophys. J. 87 (2004) 1848.

[20] G. Zgrablic, K. Voitchovsky, M. Kindermann, S. Haacke, M. Chergui, Biophys. J. 88 (2005) 2779.

[21] A. Cannizzo, O. Bram, G. Zgrablic, A. Tortschanoff, A.A. Oskouei, F. van Mourik, M. Chergui, Opt. Lett. 32 (2007) 3555.

[22] A. Cannizzo, F. van Mourik, W. Gawelda, G. Zgrablic, C. Bressler, M. Chergui, Angew. Chem. - Int. Ed. 45 (2006) 3174.

[23] W. Gawelda, A. Cannizzo, V.T. Pham, F. van Mourik, C. Bressler, M. Chergui, J. Am. Chem. Soc. 129 (2007) 8199.

[24] F.M. Jaeger, J.A. van Dijk, Z. Anorg. Allg. Chem. 227 (1936) 273.

[25] Y. Tachibana, M.K. Nazeeruddin, M. Gratzel, D.R. Klug, J.R. Durrant, Chem. Phys. 285 (2002) 127.

[26] T. Schonherr, J. Degen, E. Gallhuber, G. Hensler, H. Yersin, Chem. Phys. Lett. 158 (1989) 519.

[27] A. Hauser, Top. Curr. Chem. 234 (2004) 155

[28] H. Yersin, E. Gallhuber, G. Hensler, Chem. Phys. Lett. 134 (1987) 497.

[29] P.M. Felker, A.H. Zewail, J. Chem. Phys. 82 (1985) 2975.

[30] O. Braem, T.J. Penfold, A. Cannizzo, M. Chergui, Phys. Chem. Chem. Phys., in press.

[31] J. Gardecki, M.L. Horng, A. Papazyan, M. Maroncelli, J. Mol. Liq. 65 (6) (1995) 49.

[32] E.M. Kober, B.P. Sullivan, T.J. Meyer, Inorg. Chem. 23 (1984) 2098.

[33] S. Fantacci, F. De Angelis, A. Selloni, J. Am. Chem. Soc. 125 (2003) 4381.

[34] F. De Angelis, S. Fantacci, E. Mosconi, M.K. Nazeeruddin, M. Gratzel, J. Phys. Chem. C 115 (2011) 8825.

[35] A. Cannizzo, A.M. Blanco-Rodriguez, A. El Nahhas, J. Sebera, S. Zalis, A. Vlcek, M. Chergui, J. Am. Chem. Soc. 130 (2008) 8967.

[36] A. El Nahhas, A. Cannizzo, F. van Mourik, A.M. Blanco-Rodriguez, S. Zalis, A. Vlcek, M. Chergui, J. Phys. Chem. A 114 (2010) 6361.

[37] A.A. Oskouei, O. Bräm, A. Cannizzo, F. van Mourik, A. Tortschanoff, M. Chergui, J. Mol. Liq. 141 (2008) 118

[38] O. Bram, A.A. Oskouei, A. Tortschanoff, F. van Mourik, M. Madrid, J. Echave, A. Cannizzo, M. Chergui, J. Phys. Chem. A 114 (2010) 9034.

[39] G. Zgrablic, S. Haacke, M. Chergui, J. Phys. Chem. B 113 (2009) 4384.

[40] O. Braem, C. Consani, A. Cannizzo, M. Chergui, J. Phys. Chem. B 115 (2011) 13723.

[41] O. Braem, A. Cannizzo, M. Chergui, Ang. Chem. Int. Ed., submitted for publication. 